\title{
Approximations for the Concentration and Effectiveness Factor in Porous Catalysts of Arbitrary Shape: Taylor Series and Akbari-Ganji's Methods
}

\author{
Ramu UshaRani ${ }^{1}$, Lakshmanan Rajendran $^{1 *}$, Marwan Abukhaled ${ }^{2}$ \\ ${ }^{1}$ Department of Mathematics, AMET Deemed to be University, Chennai 603112, India \\ ${ }^{2}$ Department of Mathematics and Statistics, American University of Sharjah, Sharjah, United Arab Emirates
}

Corresponding Author Email: raj_sms@ @rediffmail.com

https://doi.org/10.18280/mmep.080405

Received: 27 February 2021

Accepted: 14 July 2021

\section{Keywords:}

mathematical modeling, nonlinear diffusion, reaction equation, Michaelis-Menten kinetic, Taylor series, Akbari-Ganji's method

\begin{abstract}
A mathematical model of reaction-diffusion problem with Michaelis-Menten kinetics in catalyst particles of arbitrary shape is investigated. Analytical expressions of the concentration of substrates are derived as functions of the Thiele modulus, the modified Sherwood number, and the Michaelis constant. A Taylor series approach and the Akbari-Ganji's method are utilized to determine the substrate concentration and the effectiveness factor. The effects of the shape factor on the concentration profiles and the effectiveness factor are discussed. In addition to their simple implementations, the proposed analytical approaches are reliable and highly accurate, as it will be shown when compared with numerical simulations.
\end{abstract}

\section{INTRODUCTION}

Nonlinear differential equations in general and reactiondiffusion equations, in particular, arise as mathematical models of various phenomena sciences and engineering, where solutions of theoretical and experimental problems are often steady-state reaction-diffusion equations with nonlinear chemical kinetics. Of these problems, we mention as examples mathematical models in immobilized enzymes $[1,2]$, microbial cells [3-5], respiring tissue [6], and artificial kidney system [7]. Various analytical and numerical approaches have been utilized to find approximate solutions to these models. For example, Tosaka and Miyake [8] analyzed a mathematical model for oxygen diffusion in a spherical cell using an integral equation method. Maalmi et al. [9] presented the mathematical expressions for the steady-state reaction rate and the associated reactant concentration profiles using semianalytical and numerical methods. Indira and Rajendran studied a mathematical model based on polyphenol oxidase catechol as a prototype enzymatic electro system [10]. Do and Greenfield used a finite integral transformation approach to solve a nonlinear kinetics problem in a general solid shape [11]. Bucolo and Tripathi studied the governing equations for the exchange of substrate between vascular and extravascular compartments on a steady-state condition [12]. Napper and Schubert investigated a mathematical Michaelis-Menten kinetics model of oxygen delivery to heart tissue [13]. Rajendran et al. used a homotopy perturbation approach for deriving an analytical expression of mediated bioelectrocatalysis concentration [14].

It is almost impossible to find exact solutions to nonlinear differential equations. Therefore, many numerical and analytical methods have been developed to find approximate solutions of nonlinear models that arise in real applications in almost all branches of sciences and engineering [15].

Although there are many effective numerical methods to solve nonlinear equations, finding approximate analytical solutions remains an ultimate goal to obtain a profound understanding of the effect of different parameters on the governing system. In addition, some serious drawbacks come along with numerical solutions, such as achieving numerical stabilities and the difficulty of adjusting parameters to match the numerical data [16]. Of the most used analytical methods, we mention the homotopy perturbation method [17, 18], homotopy analysis method [19], variational iteration method [20], Akbari-Ganji's method [21], Green's function method [22], Adomian decomposition method [23], and Taylor series method [24].

In addition to being largely accessible to researchers with a moderate mathematical background, recent applications of the Taylor series method (TSM) and Akbari-Ganji's method (AGM) have proved to be efficient and highly accurate for solving nonlinear models arise in various fields of sciences and engineering. For example, TSM has been employed in solving fractal Bratu equation [24], Lane-Emden equation [25], nonlinear reaction-diffusion equation in the electroactive polymer film [26], nonlinear equation in mass transfer [27], nonlinear concentration equation in electroanalytical chemistry [28], nonlinear reaction-diffusion problem in electrostatic interaction [29] and a nonlinear PoissonBoltzmann equations [30]. The Akbari-Ganji's method (AGM) has also been utilized for deriving semi-analytic solutions of nonlinear models. For example, Berkan [21] used AGM to study the steady three-dimensional problem of condensation film on an inclined rotating. Nirmala et al. [31] derived the steady-state substrate and product concentrations for nonMichaelis-Menten kinetics in an amperometric biosensor using the hyperbolic function method, which is a particular case of the Akbari-Ganji method. Manimegalai et al. [32] used AGM to obtain approximate analytical solutions for the nonlinear equations that describe diffusion-limited reaction within the film. Dharmalingam et al. [33] solved nonlinear 
reaction-diffusion equations that determine the substrate concentration in the electroactive polymer film using AGM. More applications of the AGM method can be found in ref. [34-36].

In the present work, we employ both the Taylor series and Akbari-Ganji's methods to investigate a nonlinear diffusion model of oxygen in general geometry. The aim is to obtain simple closed-form approximate expressions of substrate concentration and effectiveness factor using for all Thiele modulus values, Michaelis constant, and modified Sherwood numbers in a solid of general shape.

\section{MATHEMATICAL FORMULATION OF THE PROBLEM}

A cell generally consists of a surface membrane and protoplasm. In the protoplasm, some organelles compartmentalize enzymes. The sequential metabolic reactions catalyzed by these enzymes provide the necessary energy for the cells. Therefore, the oxygen, which serves as the substrate for the metabolic reactions, plays an essential role in modulating these reactions. The oxygen tension gradient in a cell even governs the distribution of different organelles. The oxygen consumption rate in cells and tissues is a complex function of oxygen tension.

The purpose of the present work is to predict the oxygen tension in a slap, cylindrical and spherical cell using an oxygen uptake kinetics of the Michaelis-Menten type. This kinetics is rigorous because it predicts reasonably well the observed oxygen uptake rates. In addition, an unsteady state oxygen diffusion model is considered here, and transients of oxygen tension may give a better understanding of the oxygen diffusion characteristics.

The particle shape is assumed to have a strong symmetry to make the composition at any point in space as a function of a single spatial variable (e.g., slab, cylinder, and spherical enzyme support). Following Lin et al. [5], the diffusion of oxygen in general geometry is expressed as follows:

$$
\begin{gathered}
D\left(\frac{d^{2} S}{d X^{2}}+\frac{n}{X} \frac{d S}{d X}\right)-\frac{V_{m} S}{S+k_{m}}=0 \\
X=0 ; \frac{d S}{d X}=0 \\
X=d ; D \frac{d S}{d X}=h\left(S_{0}-S\right)
\end{gathered}
$$

where, $\mathrm{S}$ is the concentration of oxygen, $D$ is the diffusion coefficients of oxygen, $V_{m}$ is the maximum reaction rate, $k_{m}$ is the Michaelis constant, respectively. The variable $d$ represents the radius of the cell. $S_{0}$ is the concentration of oxygen outside the cell membrane $n$ is a shape factor. By introducing the following dimensionless variables

$$
\begin{gathered}
A=\frac{S}{S_{0}}, x=\frac{X}{d}, S_{h}=\frac{h d}{D}, K_{m}=\frac{k_{m}}{S_{0}}, \\
\alpha=\left(\frac{V_{m} d^{2}}{D S_{0}}\right)
\end{gathered}
$$

Eq. (1) can be expressed in the form

$$
\frac{d^{2} A(x)}{d x^{2}}+\frac{n}{x} \frac{d A(x)}{d x}-\frac{\alpha A(x)}{K_{m}+A(x)}=0
$$

The boundary conditions are

$$
x=0, \frac{d A}{d x}=0 \text { (Symmetry condition), }
$$

$$
x=1, \frac{d A}{d x}=S_{h}(1-A) \text { (Mixed boundary condition), }
$$

where, $A$ is the dimensionless concentration of oxygen, $S_{h}$ is the modified Sherwood number, and $x$ is the radial coordinate.

The variable $n$ characterizes the immobilized catalyst shape with $n=0,1,2$ for the slab, cylindrical and spherical particle, respectively. The geometry factor can be generalized to other non-standard catalysts particles $[37,38]$. The shape parameter of the arbitrary geometry can be computed from the equation $n=\left(L S_{a} / V\right)-1$, where $L$ is the characteristic length for the chosen geometry, $S_{a}$ is the surface area of the catalyst particle, and $V$ is its volume. Non-integer values for geometries other than regular ones can also be used [39]. The effectiveness factor is given by

$$
E f=\frac{\left(1+K_{m}\right)}{\alpha}\left[\frac{d A}{d x}\right]_{x=1}
$$

From Eq. (4), it is evident that with large values of Thiele modulus $\alpha$, the rate term dominates, and the reaction is fast while slow diffusion limits the overall rate. Smaller values of the Thiele modulus represent slow reactions with rapid diffusion.

Michaelis-Menten constant $K_{m}$ reflects the enzyme's affinity for its substrate. The smaller the value of $K_{m}$, the more strongly the enzyme binds the substrate. If the $K_{m}$ value is known, it becomes possible to predict the cell needs (enzymes or substrate) to speed up the enzymatic reaction. The $K_{m}$ value approximately measures the concentration of the substrate in the cell where a reaction is occurring.

\section{CONCENTRATION OF SUBSTRATE USING THE TAYLOR SERIE METHOD (TSM)}

This section uses TSM to solve the nonlinear boundary value problem (5)-(7). As discussed in the introduction, the TSM yields a semi-analytical solution in the form of a rapidly convergent series without a need for linearization.

The analytical expression for the concentration using the TSM is given by

$$
\begin{aligned}
A(x) & =\left.\sum_{i=0}^{\infty} \frac{x^{i}}{i !} \frac{d^{i} A}{d x^{i}}\right|_{x=0} \\
& =A(0)+\left.\frac{x}{1 !} \frac{d A}{d x}\right|_{x=0}+\left.\frac{x^{2}}{2 !} \frac{d^{2} A}{d x^{2}}\right|_{x=0}+\left.\frac{x^{3}}{3 !} \frac{d^{3} A}{d x^{3}}\right|_{x=0}+
\end{aligned}
$$

The successive derivatives of the function $A(x)$ (see Appendix A) are computed at 0 and denoted by $A_{1}(0), A_{2}(0)$, $A_{3}(0)$, etc. Therefore,

$$
A(x)=A(0)+A_{1}(0) x^{2}+A_{2}(0) x^{4}+A_{3}(0) x^{6},
$$

where, 


$$
\begin{gathered}
A_{1}(0)=\frac{\alpha A(0)}{(n+1)\left(K_{m}+A(0)\right)} \frac{1}{2 !} \\
A_{2}(0)=\frac{3 \alpha^{2} A(0)}{(n+1)(n+3)\left(K_{m}+A(0)\right)^{3}} \frac{1}{4 !} \\
A_{3}(0)=\frac{15 \alpha^{3} A(0) K_{m}\left[(n+1) K_{m}-2 A(0)(n+3)\right]}{6 !(n+1)^{2}(n+3)(n+5)\left(K_{m}+A(0)\right)^{5}}
\end{gathered}
$$

From boundary condition (7), we get

$$
\begin{aligned}
& S_{h} A(0)-S_{h}+A_{1}(0)\left(2+S_{h}\right)+A_{2}(0)\left(4+S_{h}\right)+A_{3}(0) \\
& \left(6+S_{h}\right)=0
\end{aligned}
$$

The unknown quantity $A(0)$ can be obtained by solving Eq. (12). For example, for the values $S_{h}=1, K_{m}=0.5, n=0$, and $\alpha=1$, we obtain:

$$
\begin{aligned}
A(0)^{6}+3 A(0)^{5} & +3 A(0)^{4}+1.10417 A(0)^{3}-0.1125 A(0)^{2} \\
& -0.159028 A(0)-0.03125=0
\end{aligned}
$$

which leads to $A(0)=0.47119$, and hence the substrate concentration, given by Eq. (10), becomes

$$
\begin{gathered}
A(x)=0.339599+0.2022388069 x^{2}+0.1195389474 x^{4} \\
-0.0008691325663 x^{6}
\end{gathered}
$$

Using Eq. (8), the effectiveness factor is reduced to

$$
E f=\frac{\left(1+K_{m}\right)}{\alpha}\left(2 A_{1}(0)+4 A_{2}(0)+6 A_{3}(0)\right)
$$

where, $A_{1}(0), A_{2}(0)$, and $A_{3}(0)$ are given in Eq. (11).

Using the dimensionless variables in Eq. (4), the effectiveness factor is computed from Eq. (13), where

$$
K_{m}=\frac{k_{m}}{S_{0}}
$$$$
A_{1}(0)=\frac{\frac{1}{2}\left(\frac{V_{m} d^{2}}{D S_{0}}\right) A(0)}{(n+1)\left(\frac{k_{m}}{S_{0}}+A(0)\right)},
$$$$
A_{2}(0)=\frac{\frac{3}{4 !}\left(\frac{V_{m} d^{2}}{D S_{0}}\right)^{2} A(0)}{(n+1)(n+3)\left(\frac{k_{m}}{S_{0}}+A(0)\right)^{3}},
$$$$
A_{3}(0)=\frac{\frac{15}{6 !}\left(\frac{V_{m} d^{2}}{D S_{0}}\right)^{3} A(0) \frac{k_{m}}{S_{0}}\left((n+1) \frac{k_{m}}{S_{0}}-2 A(0)(n+3)\right)}{(n+1)^{2}(n+3)(n+5)\left(\frac{k_{m}}{S_{0}}+A(0)\right)^{5}} .
$$

\section{CONCENTRATION SUBSTRATE USING THE AKBARI-GANJI'S AND TAYLOR SERIES METHODS}

As discussed in the introduction, Akbari-Ganji's method is a powerful algebraic approach that produces semi-analytic approximate solutions of nonlinear differential equations. The method requires no linearization and gives solutions in terms of convergent series. The AGM begins by assuming the solution to Eq. (5) is in the form of the hyperbolic function:

$$
A(x)=B_{1} \cosh (m x)+B_{2} \sinh (m x)
$$

Substituting boundary conditions (6)-(7) in Eq. (15) gives

$$
\begin{gathered}
B_{1}=\frac{S_{h}}{m \sinh m+S_{h} \cosh m}, B_{2}=0 \\
A(x)=A(0) \cosh (m x)
\end{gathered}
$$

where, $A(0)=B_{1}=\frac{S_{h}}{m \sinh m+S_{h} \cosh m}$.

From Eq. (17), we obtain

$$
m=\cosh ^{-1}\left(\frac{A(1)}{A(0)}\right) .
$$

Therefore, a derived analytical expression of the concentration is given by

$$
A(x)=A(0) \cosh \left[x \cosh ^{-1}\left[\frac{A(1)}{A(0)}\right]\right]
$$

and the effectiveness factor is

$$
\begin{aligned}
E f & =\frac{\left(1+K_{m}\right) A(1)}{\alpha} \sinh \left[\cosh ^{-1}\left[\frac{A(1)}{A(0)}\right]\right] \\
& =\frac{\left(1+K_{m}\right) A(1)}{\alpha} \sqrt{\frac{(A(1))^{2}-A(0)^{2}}{A(0)^{2}}}
\end{aligned}
$$

As pointed out earlier, notice that $A(0)$ can be obtained from Eq. (12) for given parameters values. When $n=\frac{L S_{a}}{V}-1$, both the concentration and effectiveness factor of a substrate of arbitrary shape can be obtained from Eq. (10).

\section{PREVIOUS RESULT}

Devi et al. [40] used the Adomian decomposition method (ADM) to find the following analytical expressions for the concentration of substrate and effectiveness factor.

\subsection{Slab}

The concentration of substrate and the effectiveness factor are given by

$$
\begin{gathered}
A(x)=1+l(\alpha)+m(\alpha) x^{2} \\
-2 \frac{m(\alpha) K_{m}}{\left(K_{m}+1\right)}\left[\frac{3 l(\alpha)}{2}+\frac{5 m(\alpha)}{12}-\frac{l(\alpha) x^{2}}{2}-\frac{m(\alpha) x^{4}}{12}\right] \\
E f=\frac{\left(1+K_{m}\right)}{\alpha}\left(2 m(\alpha)+2 \frac{m(\alpha) K_{m}}{\left(K_{m}+1\right)}\left[l(\alpha)+\frac{m(\alpha)}{3}\right]\right)
\end{gathered}
$$

\subsection{Spherical}

The concentration of substrate and the effectvenes factor are given by in spherical is

$$
\begin{aligned}
& A(x)=1+\frac{1}{3}[l(\alpha)+m(\alpha)] \\
& -2 \frac{m(\alpha) K_{m}}{S_{h}\left(K_{m}+1\right)}\left[\begin{array}{l}
\frac{l(\alpha)}{9}+\frac{m(\alpha)}{15}+\frac{S_{h} l(\alpha)}{18}+\frac{S_{h} m(\alpha)}{18} \\
+\frac{S_{h} l(\alpha) x^{2}}{2}+\frac{S_{h} m(\alpha) x^{4}}{12}
\end{array}\right]
\end{aligned}
$$


where

$$
\begin{aligned}
& l(\alpha)=-\left[\frac{1}{2} \frac{\alpha}{K_{m}+1}+\frac{\alpha}{S_{h}\left(K_{m}+1\right)}\right], m(\alpha) \\
& =\frac{1}{2}\left[\frac{\alpha}{K_{m}+1}\right] . \\
& E f=\frac{\left(1+K_{m}\right)}{\alpha}\left(-2 \frac{m(\alpha) K_{m}}{S_{h}\left(K_{m}+1\right)}\left[S_{h} l(\alpha)+\frac{S_{h} m(\alpha)}{3}\right]\right)
\end{aligned}
$$

It is to be mentioned here that the Adomian decomposition method usually fails to provide any meaningful information beyond a finite interval [41].

\section{ESTIMATION OF PARAMETERS}

From Eq. (5), the reaction rate can be written in the form:

$$
\frac{1}{R}=\frac{K_{m}+A}{\alpha A}
$$

that is, $R A=\alpha A-K_{m} R$, where $K_{m}$ and $\alpha$ are unknown parameters. Using the method of least squares, the normal equation, in a matrix form can be written as

$$
\left(\begin{array}{l}
\sum A_{i}-\sum R_{i} \\
\sum A_{i} R_{i}-\sum R_{i}^{2}
\end{array}\right)\left(\begin{array}{l}
\alpha \\
K_{m}
\end{array}\right)=\left(\begin{array}{l}
\sum A_{i} R_{i} \\
\sum A_{i} R_{i}^{2}
\end{array}\right)
$$

By solving the above system, we can obtain Thiele module $(\alpha)$ and Michaela's constant $\left(K_{m}\right)$. Eq. (25) may also be written in the form

$$
\frac{1}{R}=\frac{K_{m}}{\alpha} \frac{1}{A}+\frac{1}{\alpha}
$$

By plotting $1 / R$ versus $1 / A$, we can obtain the slope $K_{m} / \alpha$ and the intercept $1 / \alpha$ and hence the kinetic parameters $K_{m}$ and $\alpha$ can be deduced.

\section{NUMERICAL SIMULATIONS}

The nonlinear differential Eq. (5) subject to boundary conditions (6) and (7) is solved numerically by using the MATLAB function "pdex4". Figure 1 shows that the analytical TSM solution (Eqs. (10)), AGM solution (Eq. (20)), ADM solution [26], and the numerical solution are in strong agreement.

For Figures 1(a)-(d), the following values of $A(0)$ were used to generate the dimensionless concentrations:

Figure 1(a): $A(0)=0.02166,0.37910,0.69631,0.98651$,

Figure 1(b): $A(0)=0.11800,0.38558,0.67532,0.99950$,

Figure 1(c): $A(0)=0.02609,0.26651,0.36167,0.46493$,

Figure $1(\mathrm{~d}): A(0)=0.26651,0.45986,0.58199$.

Tables 1-2 represent give a closer comparison between numerical results and analytical results (Taylor's series method, Akbari-Ganji's method and Adomian decomposition method). The average relative errors at discrete points show that TSM and AGM are superior to the ADM, which is valid only for small values of parameters.
Fig.1(a)

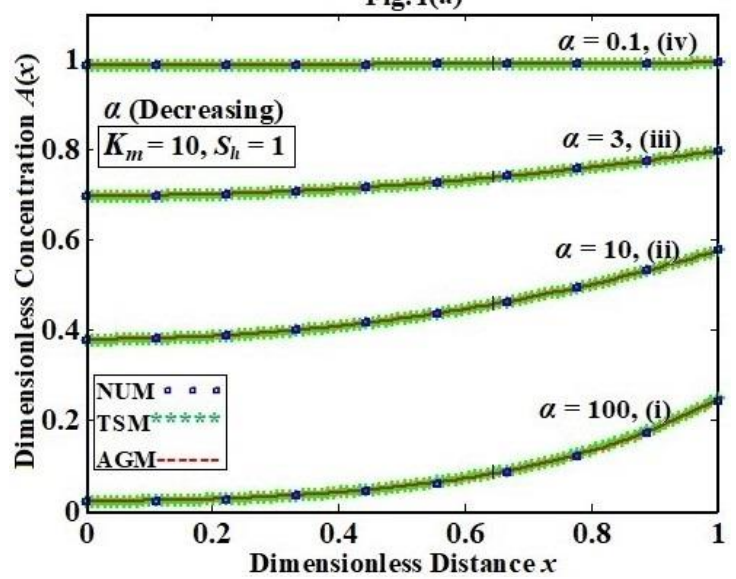

Fig.1(b)

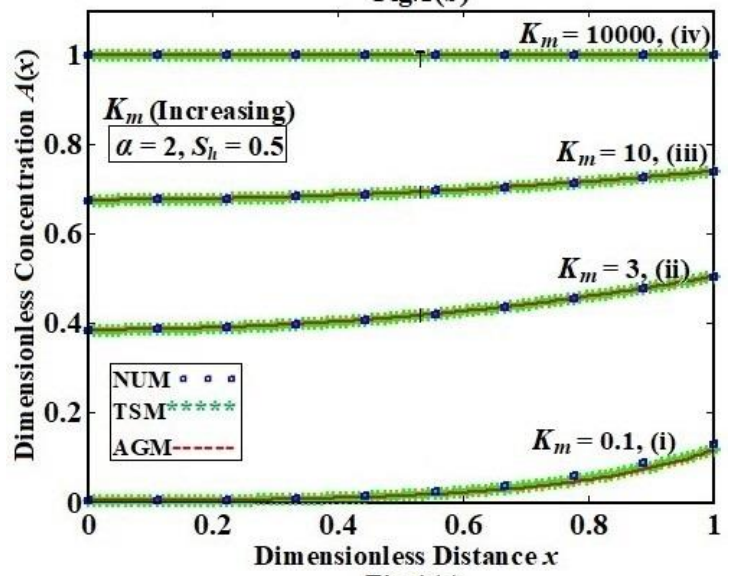

Fig.1(c)

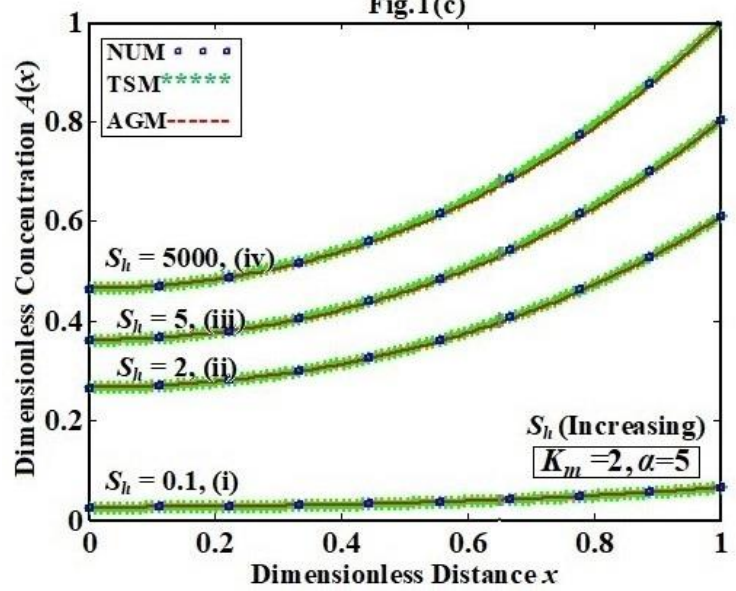

Fig. 1(d)

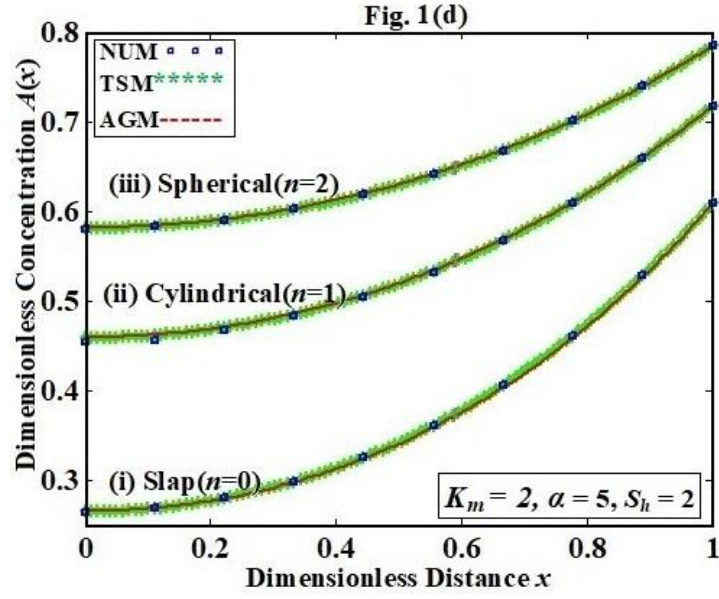

Figure 1. Dimensionless concentration substrate $A(x)$ versus dimensionless distance $x$ for different values of $A(0)$ 
Tables 3-6 show that the analytical values of the effective factor computed by the proposed TSM are equal to the numerical values for various kinetic parameters unlike the analytical values computed by the ADM where some variations are evident.

Table 1. Comparison of numerical solution of the concentration of substrate $A(x)$ with the analytical solutions by TSM, AGM, and ADM methods for $K_{m}=1, S_{h}=0.5$ and different values of $\alpha$ (Slab, $\left.n=0\right)$

\begin{tabular}{|c|c|c|c|c|c|c|c|}
\hline \multicolumn{8}{|c|}{$\alpha=0.1, A(0)=0.882322$} \\
\hline $\mathrm{x}$ & Num & TSM Eq. (10) & TSM Error (\%) & AGM Eq. (18) & AGM Error (\%) & ADM Eq.(20) & ADM Error (\%) \\
\hline 0.0 & 0.9851 & 0.9851 & 0 & 0.9851 & 0.00 & 0.9851 & 0.00 \\
\hline 0.2 & 0.9853 & 0.9853 & 0.00 & 0.9853 & 0.00 & 0.9853 & 0.00 \\
\hline 0.4 & 0.9859 & 0.9859 & 0.00 & 0.9859 & 0.00 & 0.9859 & 0.00 \\
\hline 0.6 & 0.9869 & 0.9869 & 0.00 & 0.9869 & 0.00 & 0.9869 & 0.00 \\
\hline 0.8 & 0.9883 & 0.9883 & 0.00 & 0.9883 & 0.00 & 0.9883 & 0.00 \\
\hline 1.0 & 0.9901 & 0.9901 & 0.00 & 0.9901 & 0.00 & 0.9901 & 0.00 \\
\hline \multicolumn{3}{|c|}{ Average Error } & 0.00 & & 0.00 & & 0.00 \\
\hline
\end{tabular}

$\alpha=0.2, A(0)=0.779004$

\begin{tabular}{|c|c|c|c|c|c|c|c|}
\hline $\mathrm{x}$ & Num & TSM Eq. (10) & TSM Error (\%) & AGM Eq. (18) & AGM Error (\%) & ADM Eq.(20) & ADM Error (\%) \\
\hline 0.0 & 0.6872 & 0.6872 & 0 & 0.6872 & 0.00 & 0.6888 & 0.23 \\
\hline 0.2 & 0.6914 & 0.6913 & 0.01 & 0.6913 & 0.01 & 0.6929 & 0.22 \\
\hline 0.4 & 0.7039 & 0.7036 & 0.04 & 0.7034 & 0.07 & 0.7051 & 0.17 \\
\hline 0.6 & 0.7248 & 0.7241 & 0.10 & 0.7239 & 0.12 & 0.7255 & 0.10 \\
\hline 0.8 & 0.7544 & 0.753 & 0.19 & 0.7528 & 0.21 & 0.7543 & 0.01 \\
\hline 1.0 & 0.7905 & 0.7905 & 0.00 & 0.7905 & 0.00 & 0.7917 & 0.15 \\
\hline \multicolumn{3}{|c|}{ Average Error } & 0.06 & & 0.07 & & 0.15 \\
\hline
\end{tabular}

$\alpha=0.7, A(0)=0.545345$

\begin{tabular}{|c|c|c|c|c|c|c|c|}
\hline $\mathrm{x}$ & Num & TSM Eq. (10) & TSM Error $(\%)$ & AGM Eq. (18) & AGM Error (\%) & ADM Eq.(20) & ADM Error $(\%)$ \\
\hline 0.0 & 0.4889 & 0.4889 & 0 & 0.4889 & 0.00 & 0.5101 & 4.34 \\
\hline 0.2 & 0.4954 & 0.4953 & 0.02 & 0.4952 & 0.04 & 0.5163 & 4.22 \\
\hline 0.4 & 0.5153 & 0.5147 & 0.12 & 0.5143 & 0.19 & 0.535 & 3.82 \\
\hline 0.6 & 0.5487 & 0.5475 & 0.22 & 0.5468 & 0.35 & 0.5665 & 3.24 \\
\hline 0.8 & 0.5964 & 0.5942 & 0.37 & 0.5935 & 0.49 & 0.6113 & 2.50 \\
\hline 1.0 & 0.6555 & 0.6555 & 0.00 & 0.6555 & 0.00 & 0.6701 & 2.23 \\
\hline \multicolumn{3}{|c|}{ Average Error } & 0.12 & & 0.18 & & 3.39 \\
\hline
\end{tabular}

Table 2. Comparison of numerical solution of the concentration of substrate $A(x)$ with the analytical solutions by TSM, AGM, and $\mathrm{ADM}$ for $K_{m}=1, \alpha=0.5$ for different values of $S_{h}$ (Spherical, $n=2$ )

\begin{tabular}{|c|c|c|c|c|c|c|c|}
\hline \multicolumn{8}{|c|}{$\mathrm{S}_{\mathrm{h}}=1, \mathrm{~m}=0.881506$} \\
\hline $\mathrm{x}$ & Num & $\begin{array}{c}\text { TSM Eq. } \\
(10)\end{array}$ & $\begin{array}{c}\text { TSM Error } \\
(\%)\end{array}$ & AGM Eq. (18) & $\begin{array}{c}\text { AGM Error } \\
(\%)\end{array}$ & ADM Eq.(22) & $\begin{array}{c}\text { ADM Error } \\
(\%)\end{array}$ \\
\hline 0.0 & 0.8815 & 0.8815 & 0 & 0.8815 & 0.00 & 0.9232 & 4.73 \\
\hline 0.2 & 0.8831 & 0.8831 & 0.00 & 0.8831 & 0.00 & 0.9233 & 4.55 \\
\hline 0.4 & 0.8879 & 0.8876 & 0.03 & 0.8877 & 0.02 & 0.9236 & 4.02 \\
\hline 0.6 & 0.8959 & 0.8956 & 0.03 & 0.8956 & 0.03 & 0.9241 & 3.15 \\
\hline 0.8 & 0.9071 & 0.9066 & 0.06 & 0.9066 & 0.06 & 0.9547 & 5.25 \\
\hline 1.0 & 0.9208 & 0.9208 & 0.00 & 0.9208 & 0.00 & 0.9255 & 0.51 \\
\hline \multicolumn{3}{|c|}{ Average Error } & 0.02 & & 0.02 & & 3.70 \\
\hline \multicolumn{8}{|c|}{$\mathrm{S}_{\mathrm{h}}=2, \mathrm{~m}=0.919362$} \\
\hline $\mathrm{x}$ & Num & $\begin{array}{c}\text { TSM Eq. } \\
\text { (10) }\end{array}$ & $\begin{array}{c}\text { TSM Error } \\
(\%)\end{array}$ & AGM Eq. (18) & $\begin{array}{c}\text { AGM Error } \\
(\%)\end{array}$ & ADM Eq.(22) & DM Error $(\%)$ \\
\hline 0.0 & 0.9194 & 0.9194 & 0 & 0.9194 & 0.00 & 0.961 & 4.52 \\
\hline 0.2 & 0.921 & 0.921 & 0.00 & 0.921 & 0.00 & 0.9611 & 4.35 \\
\hline 0.4 & 0.9259 & 0.9258 & 0.01 & 0.9258 & 0.01 & 0.9613 & 3.82 \\
\hline 0.6 & 0.9341 & 0.9338 & 0.03 & 0.9338 & 0.03 & 0.9616 & 2.94 \\
\hline 0.8 & 0.9455 & 0.945 & 0.05 & 0.945 & 0.05 & 0.962 & 1.75 \\
\hline 1.0 & 0.9595 & 0.9595 & 0.00 & 0.9595 & 0.00 & 0.9625 & 0.31 \\
\hline \multicolumn{3}{|c|}{ Average Error } & 0.02 & & 0.02 & & 2.95 \\
\hline \multicolumn{8}{|c|}{$\mathrm{S}_{\mathrm{h}}=3, \mathrm{~m}=0.932364$} \\
\hline $\mathrm{x}$ & Num & $\begin{array}{c}\text { TSM Eq. } \\
(10)\end{array}$ & $\begin{array}{c}\text { TSM Error } \\
(\%)\end{array}$ & AGM Eq. (18) & $\begin{array}{c}\text { AGM Error } \\
(\%)\end{array}$ & ADM Eq.(22) & $\begin{array}{c}\text { ADM Error } \\
(\%)\end{array}$ \\
\hline 0.0 & 0.9324 & 0.9324 & 0 & 0.9324 & 0.00 & 0.974 & 4.46 \\
\hline 0.2 & 0.934 & 0.934 & 0.00 & 0.934 & 0.00 & 0.9741 & 4.29 \\
\hline 0.4 & 0.9389 & 0.9388 & 0.01 & 0.9388 & 0.01 & 0.9743 & 3.77 \\
\hline 0.6 & 0.9472 & 0.9469 & 0.03 & 0.9469 & 0.03 & 0.9745 & 2.88 \\
\hline 0.8 & 0.9587 & 0.9582 & 0.05 & 0.9582 & 0.05 & 0.9749 & 1.69 \\
\hline 1.0 & 0.9728 & 0.9728 & 0.00 & 0.9728 & 0.00 & 0.9752 & 0.25 \\
\hline \multicolumn{3}{|c|}{ Average Error } & 0.02 & & 0.02 & & 2.89 \\
\hline
\end{tabular}


Table 3. Comparison between simulation results and analytical results (TSM and ADM) for effective factor with fixed values of $K_{m}=0.1$ and $S_{h}=5$ and various values $\alpha$

\begin{tabular}{cccccc}
\hline $\boldsymbol{\alpha}$ & Num & TSM Eq. (13) & \% TSM Error & ADM Eq. (21) & \% ADM Error \\
\hline 0 & 0.995 & 0.995 & 0.00 & 0.9952 & 0.02 \\
0.1 & 0.9954 & 0.9954 & 0.00 & 0.9956 & 0.02 \\
0.2 & 0.9904 & 0.9904 & 0.00 & 0.9912 & 0.08 \\
0.3 & 0.9848 & 0.9848 & 0.00 & 0.9868 & 0.20 \\
0.4 & 0.9787 & 0.9787 & 0.00 & 0.9824 & 0.38 \\
0.5 & 0.9718 & 0.9718 & 0.00 & 0.978 & 0.64 \\
0.6 & 0.9641 & 0.9641 & 0.00 & 0.9736 & 0.99 \\
0.7 & 0.9553 & 0.9553 & 0.00 & 0.9691 & 1.44 \\
0.8 & 0.9451 & 0.9451 & 0.00 & 0.9647 & 2.07 \\
0.9 & 0.9331 & 0.9331 & 0.00 & 0.9603 & 2.92 \\
1 & 0.9188 & 0.9188 & 0.00 & 0.9559 & 4.04 \\
\hline Avg. Error & \multicolumn{5}{c}{$\mathbf{0 . 0 0}$} \\
\hline
\end{tabular}

Table 4. Comparison between simulation results and analytical results (TSM and ADM) for effective factor with fixed values of $K_{m}=50$ and $S_{h}=5$ and various values $\alpha$

\begin{tabular}{cccccc}
\hline $\boldsymbol{\alpha}$ & Num & TSM Eq. (13) & \% TSM Error & ADM Eq. (21) & \% ADM Error \\
\hline 0 & 0.9995 & 0.9995 & 0.00 & 0.9995 & 0.00 \\
0.1 & 0.999 & 0.999 & 0.00 & 0.999 & 0.00 \\
0.2 & 0.998 & 0.998 & 0.00 & 0.998 & 0.00 \\
0.3 & 0.9969 & 0.9969 & 0.00 & 0.9969 & 0.00 \\
0.4 & 0.9959 & 0.9959 & 0.00 & 0.9959 & 0.00 \\
0.5 & 0.9948 & 0.9948 & 0.00 & 0.9948 & 0.00 \\
0.6 & 0.9939 & 0.9939 & 0.00 & 0.9939 & 0.00 \\
0.7 & 0.9929 & 0.9929 & 0.00 & 0.9929 & 0.00 \\
0.8 & 0.9919 & 0.9919 & 0.00 & 0.9919 & 0.00 \\
0.9 & 0.9909 & 0.9909 & 0.00 & 0.9909 & 0.00 \\
1 & 0.9899 & 0.9899 & 0.00 & 0.9899 & 0.00 \\
\hline Avg. Error & \multicolumn{5}{c}{}
\end{tabular}

Table 5. Comparison between simulation results and analytical results (TSM and ADM) for effective factor with fixed values of $\alpha=0.1$ and $S_{h}=5$ and various values $K_{m}$

\begin{tabular}{cccccc}
\hline $\mathbf{K}_{\mathbf{m}}$ & Num & TSM Eq. (13) & \% TSM Error & ADM Eq. (21) & \% ADM Error \\
\hline 0 & 0.995 & 0.995 & 0.00 & 0.995 & 0.00 \\
0.1 & 0.9954 & 0.9954 & 0.00 & 0.9954 & 0.00 \\
0.2 & 0.9923 & 0.9923 & 0.00 & 0.9923 & 0.00 \\
0.3 & 0.9903 & 0.9903 & 0.00 & 0.9903 & 0.00 \\
0.4 & 0.9889 & 0.9889 & 0.00 & 0.9889 & 0.00 \\
0.5 & 0.988 & 0.988 & 0.00 & 0.988 & 0.00 \\
0.6 & 0.9874 & 0.9874 & 0.00 & 0.9874 & 0.00 \\
0.7 & 0.987 & 0.987 & 0.00 & 0.987 & 0.00 \\
0.8 & 0.9868 & 0.9868 & 0.00 & 0.9868 & 0.00 \\
0.9 & 0.9867 & 0.9867 & 0.00 & 0.9867 & 0.00 \\
1 & 0.9867 & 0.9867 & 0.00 & 0.9867 & 0.00 \\
\hline Avg. Error & \multicolumn{5}{c}{}
\end{tabular}

Table 6. Comparison between simulation results and analytical results (TSM and ADM) for effective factor with fixed values of $\alpha=1$ and $S_{h}=5$ and various values $K_{m}$

\begin{tabular}{cccccc}
\hline $\mathbf{K}_{\mathbf{m}}$ & Num & TSM Eq. (13) & \% TSM Error & ADM Eq. (21) & \% ADM Error \\
\hline 0 & 0.92 & 0.92 & 0.00 & 0.9559 & 3.90 \\
0.1 & 0.9188 & 0.9188 & 0.00 & 0.9359 & 1.86 \\
0.2 & 0.8933 & 0.8933 & 0.00 & 0.9259 & 3.65 \\
0.3 & 0.8799 & 0.8799 & 0.00 & 0.9053 & 2.89 \\
0.4 & 0.8726 & 0.8726 & 0.00 & 0.8912 & 2.13 \\
0.5 & 0.8705 & 0.8705 & 0.00 & 0.8815 & 1.26 \\
0.6 & 0.8685 & 0.8685 & 0.00 & 0.8750 & 0.75 \\
0.7 & 0.8670 & 0.8670 & 0.00 & 0.8708 & 0.44 \\
0.8 & 0.8655 & 0.8655 & 0.00 & 0.8683 & 0.32 \\
0.9 & 0.8640 & 0.8640 & 0.00 & 0.867 & 0.35 \\
1 & 0.8620 & 0.8620 & 0.00 & 0.8667 & 0.55 \\
\hline Avg. Error & \multicolumn{5}{c}{} \\
\hline
\end{tabular}




\section{RESULTS AND DISCUSSION}

The nonlinear Eq. (5) is solved using two simple, efficient, and reliable approaches: The Taylor series and the AkbariGanji methods. Semi-analytical approximate concentrations were obtained for all values of parameters using Taylor series (Eq. (10)) and AGM (Eq. (18)). Figures 1 and 2 show that there is a strong agreement between the analytical and numerical results. From Tables 1 and 2, it is confirmed that the accuracy of the proposed methods (TSM and AGM) significantly surpasses the accuracy of AGM.

The obtained concentration depends on the Michaelis constant $\left(K_{m}\right)$, Thiele module $(\alpha)$, and the modified Sherwood number $\left(S_{h}\right)$. The Thiele modulus, which is used for the determination of the effectiveness factor, describes the relationship between diffusion and reaction as follows:

$$
\alpha=\sqrt{\frac{V_{m} L^{2}}{2 D S_{0}}}=\frac{\text { Diffusion Time }}{\text { Reaction Time }}
$$

The Sherwood number $S_{h}$, which is also known as the mass transfer Nusselt number represents the ratio of convective to diffusive mass transfer coefficients, that is

$$
S_{h}=\frac{K_{m} L}{D}=\frac{\text { Convective mass transfer coefficient }}{\text { Diffusion mass transfer coefficient }}
$$

where, $L$ is a characteristic length, $D$ is the mass diffusivity, $k_{m}$ is the mass transfer coefficient. Figure 1 represents the substrate's concentration for various Michaelis-Menten values of the constant $K_{m}$, Thiele modulus $\alpha$, and Sherwood number $S_{h}$ for planar, cylindrical, and spherical particles. It is inferred from Figure 1 that the concentration of the substrate increases when Michaelis's constant $\left(K_{m}\right)$ and Sherwood number $\left(S_{h}\right)$ increase or the Thiele modulus $(\alpha)$ decreases for all geometries. The concentration curves for the spherical, cylindrical, and planar particles are plotted in Figure 1(d). It is observed that the substrate concentration for a spherical particle is greater than that of a planar or a cylindrical particle.

To measure variations in reaction rates throughout the process, a parameter for the effectiveness factor, which is the ratio of the pellet's overall reaction rate to the reaction rate at the pellet's external surface, is introduced.

The effectiveness factor is an essential indicator of pore diffusion and reactions on pore walls in porous catalytic pellets and solid fuel particles. It is established by Thiele [35] that the reaction rate in a particle may be expressed by the product of its rate under surface conditions and the effectiveness factor.

Figure 2 demonstrates the effects of Thiele modulus, Michaelis constant, modified Sherwood number, and shape factors (slap, cylindrical and spherical) on the effectiveness factor. When the reaction rate controls the process, the effectiveness factor approaches unity. However, the effectiveness factor decreases rapidly when Thiele modulus's value increases (Figures 2(a)-2(c)) for all other parameters' values.

At high Sherwood number values $\left(S_{h}\right)$, the mass transfer limitations are the dominating factors in determining the substrate conversion. Figure 2(a) shows the effects of $S_{h}$ on the internal effectiveness factor. The effectiveness factor continues to increase slowly as $S_{h}$ increases. When $S_{h}$, however, becomes larger than 1000 , the effectiveness factor becomes independent of it.
From Figure 2(b), it is inferred that the effectiveness factor is uniform for high Michaelis constant due to the intraparticle diffusion.
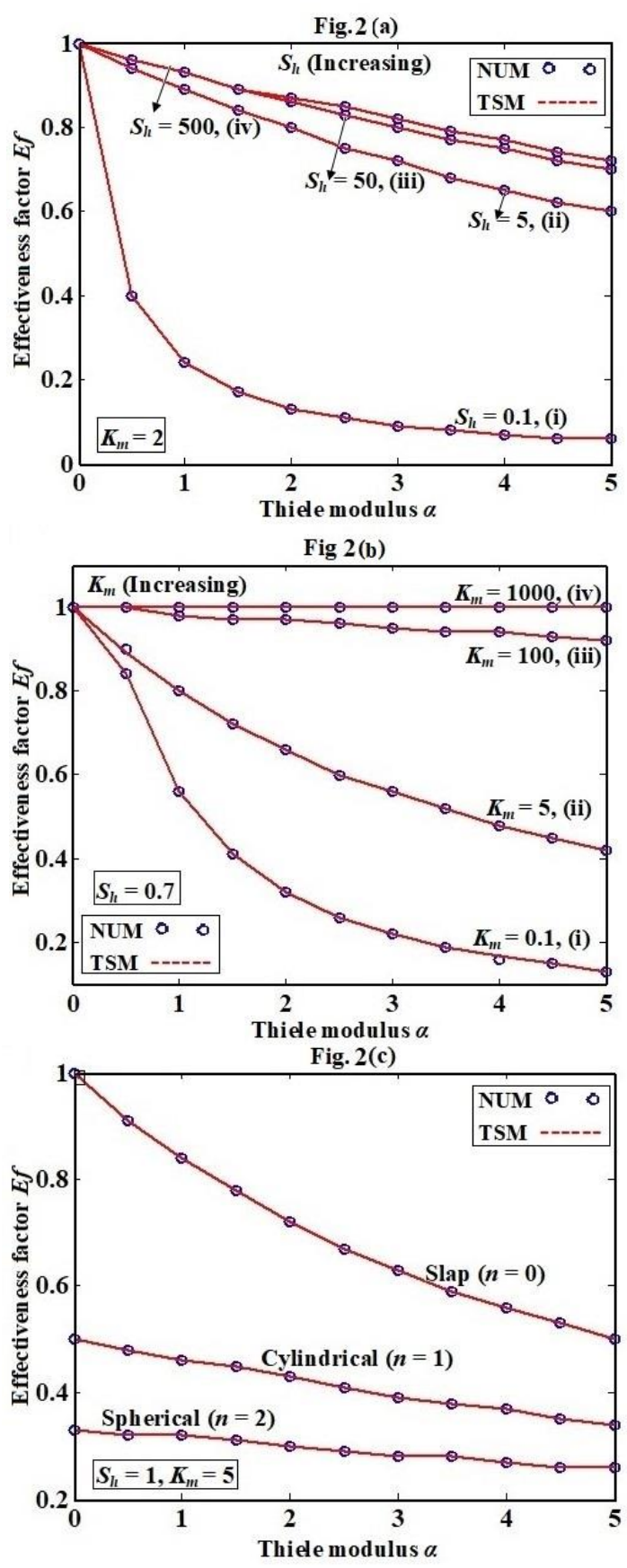

Figure 2. Comparison of analytical expression of effective factor with simulation results for various values of $K_{m}, \alpha$ and $S_{h}$

The effectiveness factor curves for the spherical, cylindrical, and slap particles are plotted in Figure 2(c). From these figures, it is observed that the effectiveness factor for slap particle is more significant than a planar, cylindrical, and spherical particle.

Figures 3(a)-(b) illustrate the influence of shape factor on the concentration and effectiveness factor for various parameters' values. From these figures, it is inferred that slab shape gave better results on the effectiveness factor than those of the other shapes. A better diffusion mass transfer in the slab shape leads to a better yield.

The limiting cases for saturated (zero-order) catalytic 
kinetics and un-saturated (first-order) catalytic kinetics are discussed in Appendix B.

Fig. 3(a)

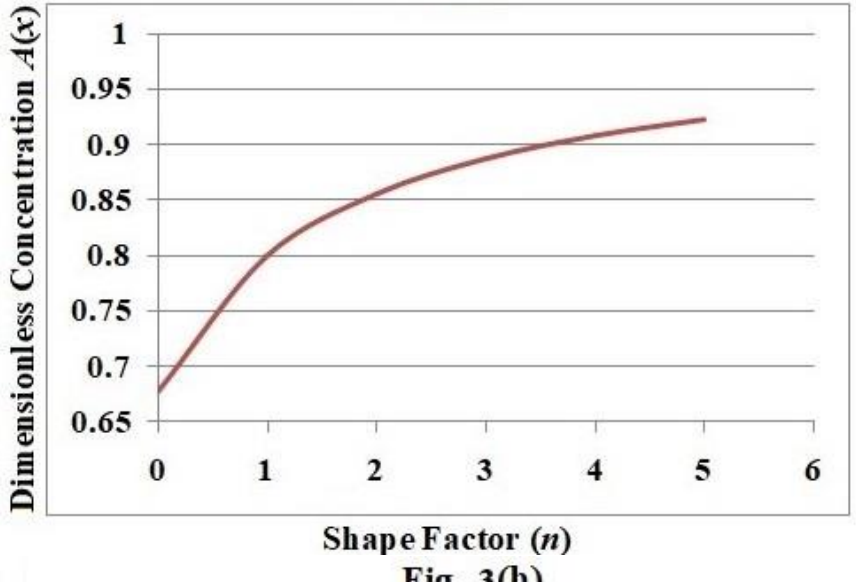

Fig. 3(b)

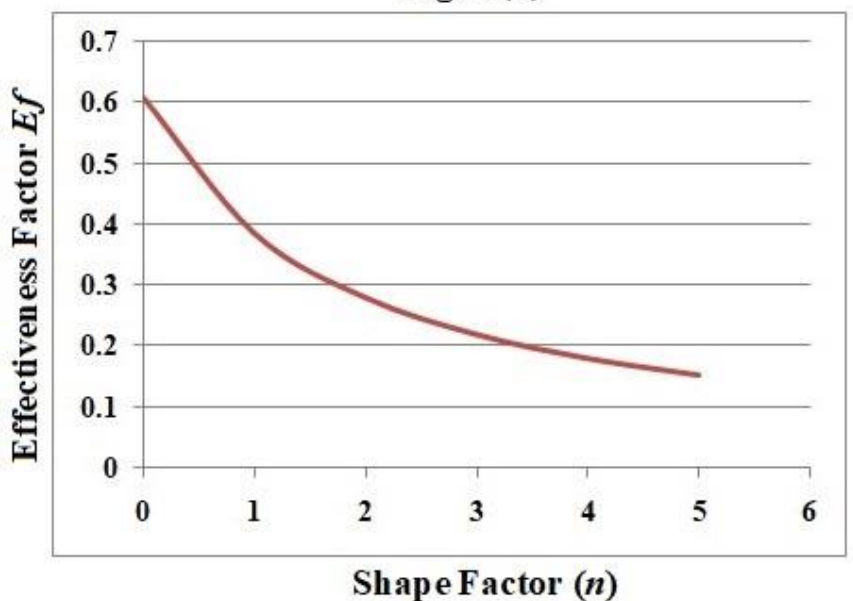

Figure 3. Concentration of substrate and effectiveness factor versus shape factor

\section{CONCLUSION}

Two approximate semi-analytical methods for solving reaction-diffusion problems inside catalyst particles were discussed. Analytical expressions for the substrate's concentration and the effectiveness factor for all parameters are obtained using Taylor series and Akbari-Ganji's methods. The effects of Michaelis constant, Thiele module, and modified Sherwood number, and the shape factor on the concentration profiles were discussed. The sensitivity analysis and estimation of the kinetics parameter were also reported. It was concluded that a lower shape factor results in a stronger effectiveness factor. In terms of mass diffusion transfer, the other form is inferior to the slab shape, leading to a lower yield. The analytical results derived by the proposed methods produced less relative error than the results of the Adomian decomposition method when compared to numerical simulation results. The proposed approximation approach can be applied to several multicomponent reactions in various catalyst geometries with adequate precision.

\section{ACKNOWLEDGMENT}

This work was supported by the Academy of Maritime
Education and Training (AMET), Deemed to be University, Chennai. The authors are thankful to Shri J. Ramachandran, Chancellor, Col. and Dr. G. Thiruvasagam, Vice-Chancellor of AMET, Chennai, Tamil Nadu, for their immense support.

\section{REFERENCES}

[1] Aris, R. (1975). The Mathematical theory of diffusion and reaction in permeable. catalysts. Clarendon Oxford, 21(5): 444-460. https://doi.org/10.1002/aic.690210537

[2] Engasser, J.M., Horvath, C. (1973). Effect of internal diffusion in heterogeneous enzyme systems: Evolution of true kinetic parameters and substrate diffusity. Journal of Theoretical Biology, 42(1): 137-155. https://doi.org/10.1016/0022-5193(73)90153-7

[3] Chevillotte, P. (1973). Relation between the reaction cytochrome oxidase-oxygen and oxygen uptake in cells in vivo. Journal of Theoretical Biology, 39(2): 277-295. https://doi.org/10.1016/0022-5193(73)90098-2

[4] McElwain, D.L.S. (1978). A re-examination of oxygen diffusion in a spherical cell with michaelis-menten oxygen uptake kinetics. Journal of Theoretical Biology, 71(2): 255-263. https://doi.org/10.1016/0022193(78)90270-9

[5] Lin, S.H. (1976). Oxygen diffusion in a spherical cell with nonlinear oxygen uptake kinetics. Journal of Theoretical Biology, 60(2): 449-457. https://doi.org/10.1016/0022-5193(76)90071-0

[6] Stroeve, P. (1997). Diffusion with irreversible chemical reaction in heterogeneous media; Application to oxygen transport in respiring tissue. Journal of Theoretical Biology, 64: 237-251. https://doi.org/10.1016/00225193(77)90354-X

[7] Lin, S.H. (1979). A modified model for predicting the performance of a compact artificial kidney. Journal of Theoretical Biology, 77: 441-451. https://doi.org/10.1016/0022-5193(79)90019-5

[8] Tosaka, N., Miyake, S. (1982). Analysis of a nonlinear diffusion problem With Michaelis-Menten kinetics by an integral equation method. Bulletin of Mathematical Biology, 44: 841-849. https://doi.org/10.1007/BF02465184

[9] Maalmi, M., Strieder, W., Varma, A. (2001). Ligand diffusion and receptor mediated internalization: Michaelis-Menten kinetics. Chemical Engineering Science. 56(19): 5609-5616. https://doi.org/10.1016/S0009-2509(01)00167-1

[10] Indira, K., Rajendran, L. (2011). Analytical expression of the concentration of substrates and product in phenolpolyphenol oxidase system immobilized in laponite ${ }^{\circledR}$ hydrogels. Michaelis-Menten formalism in homogeneous medium. Electrochimica. Acta, 56(18): 6411-6419. https://doi.org/10.1016/j.electacta.2011.05.012

[11] Do, D.D., Greenfield, P.F. (1981). A finite integral transform technique for solving the diffusion-reaction equation with Michaelis-Menten kinetics. Mathematical Biosciences, 54(1-2): 31-47. https://doi.org/10.1016/0025-5564(81)90074-2

[12] Bucolo, R.J., Tripathi, V.K. (1980). Steady-state analysis of a two-compartment barrier-limited capillary-tissue model with michaelis-menten saturation kinetics. Bulletin of Mathematical Biology, 42(5): 691-700. 
https://doi.org/10.1007/BF02460987

[13] Napper, A., Schubert, W. (1981). Michaelis-Menten kinetics as a modeling assumption in a model of oxygen transport in heart. Proceedings of the first southern. Biomedical Engineering Conference, pp. 201-204. https://doi.org/10.1016/B978-0-08-028826-0.50047-0

[14] Rajendran, L., Saravanakumar, K. (2014). Analytical expression of transient and steady-state catalytic current of mediated bioelectrocatalysis. Electrochimica Acta. 147: 678-687. https://doi.org/10.1016/j.electacta.2014.08.126

[15] Rajendran, L., Swaminathan, R., Chithra Devi, M. (2020). A Closer Look at a Nonlinear Reaction Diffusion Equation. NOVA Publishers.

[16] Chitra Devi, M., Pirabaharan, P., Rajendran, L., Abukhaled, M. (2020). An efficient method for finding analytical expressions of substrate concentrations for different particles in an immobilized enzyme system. Reaction Kinetics, Mechanisms and Catalysis, 130: 3553. https://doi.org/10.1007/s11144-020-01757-0

[17] He, J.H. (1999). Homotopy perturbation technique. Comput. Methods Appl. Mech. Engrg., 178: 257-262.

[18] Selvi, M.S.M., Rajendran, L., Abukhaled, M. (2021). Estimation of rolling motion of ship in random beam seas by efficient analytical and numerical approaches. J. Marine. Sci. Appl., 20: 55-66. https://doi.org/10.1007/s11804-020-00183-x

[19] Liao. S.J. (2009). Notes on the homotopy analysis method: some definitions and theorems. Commun. Nonlinear Sci. Numer. Simul., 14: 983-997.

[20] Rajendran. L., Rahamathunissa. G. (2008). The Application of He's variational iteration method to nonlinear boundary value problems in enzyme-substrate reaction diffusion processes: Part 1 . The steady-state amperometric response, Mathematical Chemistry, 44: 849-861. https://doi.org/10.1007/s10910-007-9340-9

[21] Berkan, S., Hoseini, S.R., Ganji, D.D. (2017). Analytical investigation of steady three-dimensional problem of condensation film on inclined rotating disk by AkbariGanji's method. J. Propuls. Power, 6(4): 277-284. https://doi.org/10.1016/j.jppr.2016.08.001

[22] Abukhaled, M., Khuri, S. (2021). A fast convergent semi-analytic method for an electrohydrodynamic flow in a circular cylindrical conduit. Int. J. Appl. Comput. Math, 7: 32. https://doi.org/10.1007/s40819-021-00974$\mathrm{y}$

[23] Anitha, A., Loghambal, S., Rajendran, L. (2012). Analytical expressions for steady-state concentrations of substrate and product in an amperometric biosensor with the substrate inhibition-the Adomian decomposition method. American J. Analytical Chemistry, 3(8): 495502. https://doi.org/10.4236/ajac.2012.38066

[24] He, C.H., Shen, Y., Ji, F.Y., He, J.H. (2019). Taylor series solution for fractal Bratu-type equation arising in electrospinning process. Fractals, 28(1). https://doi.org/10.1142/S0218348X20500115

[25] He, J.H., Ji, F.Y. (2019). Taylor series solution for LaneEmden equation. J. Math Chem., 57(2-3): 1932-1934. https://doi.org/10.1007/s10910-019-01048-7

[26] Usha Rani, R., Rajendran, L. (2020). Taylor's series method for solving the nonlinear reaction-diffusion equation in the electroactive polymer film. Chemical Physics Letters, 754(8): 137573. https://doi.org/10.1016/j.cplett.2020.137573
[27] Lilly Clarance Mary, Usha Rani, R., Meena, A., Rajendran, L. (2021). Nonlinear mass transfer at the electrodes with reversible homogeneous; reactions: Taylor's series and Hyperbolic Function Method. Int. J. Electrochemical Sciences, 16: 151037.

[28] Usha Rani, R., Rajendran, L., Lyons, M.E.G. (2021). Steady-State current in product inhibition kinetics in an amperometric biosensor: Adomian decomposition and Taylor series method. Electroanalytical Chemistry, 886: 115103.

[29] Sylvia, S.V., Salomi, R.J., Rajendran, L., Abukhaled, M. (2021). Solving nonlinear reaction-diffusion problem in electrostatic interaction with reaction-generated $\mathrm{pH}$ change on the kinetics of immobilized enzyme systems using Taylor series method. Mathematical Chemistry, 59(5): 1332-1347. https://doi.org/10.1007/s10910-02101241-7

[30] Vinolyn Sylvia, S., Joy Salomi, R., Rajendran, L., Abukhaled, M. (2020). Poisson-Boltzmann equation and electrostatic potential around macroions in colloidal plasmas: Taylor series approach. Solid State Technology, 63(6): 10090-10106.

[31] Nirmala, K.B., Manimegalai, B., Rajendran,L. (2020). Steady-State substrate and product concentrations for non-michaelis-menten kinetics in an amperometric biosensor-hyperbolic function and padéapproximants method. Int. J. Electrochemical Sciences, 15: 5682-5697.

[32] Manimegalai, B., Lyons, M.E.G., Rajendran, L. (2021). A kinetic model for amperometric immobilized enzymes at planar, cylindrical and spherical electrodes: The Akbari-Ganji method. Electroanalytical Chemistry, 880(2):

114921. https://doi.org/10.1016/j.jelechem.2020.11492

[33] Dharmalingam, K.M., Veeramuni, M. (2019). AkbariGanji's Method (AGM) for solving non-linear reaction Diffusion equation in the electroactive polymer film. Electroanalytical Chemistry, 844. https://doi.org/10.1016/j.jelechem.2019.04.061

[34] Akbari, M.R., Ganji, D.D., Rostami, A.K., Nimafar, M. (2015). Solving nonlinear differential equation governing on the rigid beams on viscoelastic foundation by AGM. Journal of Marine Science and Application, 14: 30-38. https://doi.org/10.1007/s1 1 804-015-1284-z

[35] Meresht, N.B., Ganji, D.D. (2016). Solving Nonlinear Differential Equation Arising in Dynamical Systems by AGM. Int. J. Applied Computational Mathematics, 3(2): 1507-1523. https://doi.org/10.1007/s40819-015-0131-X

[36] SheikholeslamiM., Jalili, P., Ganji, D.D. (2017). Magnetic field effect on nanofluid flow between two circular cylinders using AGM. Alexandria Engineering, 57(2): 587-594. https://doi.org/10.1016/j.aej.2017.02.01

[37] Burghardt, A., Kubaczka, A. (1996). Generalization of the effectiveness factor for any shape of a catalyst pellet. Chemical Engineering Process, 35: 65-74.

[38] Alopaeus, V. (2019). Approximating catalyst effectiveness factors with reaction rate profiles. Catalysts, 255: 1-16. https://doi.org/10.3390/catal9030255

[39] Mariani, N.J., Mocciaro, C., Keegan, S.D., Martinez, O.M., Barreto, G.F. (2009). Evaluating the effectiveness factor from a 1D approximation fitted at high Thiele modulus: Spanning commercial pellet shapes with linear kinetics. Chemical Engineering Science, 64: 2762-2766. https://doi.org/10.1016/j.ces.2009.02.044

[40] Renuga Devi, M., Sevukaperumal, S., Rajendran, L. 
(2015). Non-linear reaction diffusion equation with michaelis-menten kinetics and adomian decomposition method. Applied Mathematics, 5(1): 21-32. https://doi.org/10.5923/j.am.20150501.04

[41] Alhuthali, M. (2008). Limitations of Adomian decomposition and homotopy methods. International J. Pure and Applied Mathematics, 46: 251-260.

\section{NOMENCLATURE}

\begin{tabular}{|c|c|c|}
\hline Symbols & Name & Unit \\
\hline$S$ & Substrate concentration & $\mathrm{mol} / \mathrm{cm}^{3}$ \\
\hline$S_{0}$ & Bulk-substrate concentration & $\mathrm{mol} / \mathrm{cm}^{3}$ \\
\hline$k_{m}$ & Michaelis - Menten constant & $\mathrm{mol} / \mathrm{cm}^{3}$ \\
\hline$D$ & $\begin{array}{l}\text { Effective diffusivity inside the } \\
\text { particle }\end{array}$ & $\mathrm{cm}^{2} \mathrm{~s}^{-1}$ \\
\hline$V_{m}$ & Maximum reaction rate & $\mathrm{mol} / \mathrm{cm}^{3} \mathrm{~s}$ \\
\hline$x$ & Spatial variable & $\mathrm{cm}$ \\
\hline$h$ & Permeability of the membrane at $X=d$ & $\mathrm{~cm} / \mathrm{s}$ \\
\hline$d$ & Radius of the cell & $\mathrm{cm}$ \\
\hline$\alpha$ & Dimensionless reaction rate & \\
\hline$X$ & Dimensionless Spatial Variable & \\
\hline$K_{m}$ & $\begin{array}{l}\text { Dimensionless Michaelis -Menten } \\
\text { constant }\end{array}$ & \\
\hline$S_{h}$ & Modified Sherwood number & \\
\hline$A$ & $\begin{array}{l}\text { Dimensionless substrate } \\
\text { concentration }\left(=S / S_{0}\right)\end{array}$ & \\
\hline$n$ & $\begin{array}{l}\text { Shape factor ( } n=0 \text { for slab, } n=1 \text { for } \\
\text { cylindrical and } n=2 \text { for spherical } \\
\text { particle, } n=\left(L S_{a} / V\right)-1 \text { for general } \\
\text { geometry), } L \text { is the characteristic } \\
\text { length for the chosen geometry, } S_{a} \text { is } \\
\text { the surface area of the catalyst } \\
\text { particle, and } V \text { is the volume }\end{array}$ & \\
\hline
\end{tabular}

\section{APPENDIX A. APPROXIMATE ANALYTICAL SOLUTION OF EQ. (5) USING TAYLOR SERIES}

The Taylor series solution of Eq. (5) is given in the form

$$
A(x)=A(0)+A^{\prime}(0) \frac{x}{1 !}+A^{\prime \prime}(0) \frac{x^{2}}{2 !}+\cdots
$$

From the boundary condition Eq. (6), we have

$$
A^{\prime}(0)=0
$$

and Eq. (5) can be rewritten as follows:

$$
\left[x A^{\prime \prime}(x)+n A^{\prime}(x)\right]\left(K_{m}+A(x)\right)-x \alpha A(x)=0
$$

Differentiate the above equation with respect to $x$ we get

$$
\begin{aligned}
& {\left[x A^{\prime \prime \prime}(x)+(n+1) A^{\prime \prime}(x)\right]\left(K_{m}+A(x)\right)} \\
& =-A^{\prime}(x)\left[n A^{\prime}(x)+x A^{\prime \prime}(x)\right]+\alpha x A^{\prime}(x)+\alpha A(x)
\end{aligned}
$$

When $x=0$, Eq. (A4) results in

$$
\left[(n+1) A^{\prime \prime}(0)\right]\left(K_{m}+A(0)\right)=\alpha A(0),
$$

and hence

$$
A^{\prime \prime}(0)=\frac{\alpha A(0)}{(n+1)\left(K_{m}+A(0)\right)}
$$

Differentiating Eq. (A7) with respect to $x$ and substituting $x=0$ gives

$$
\begin{aligned}
& (n+2) A^{\prime \prime \prime}(0)\left(K_{m}+A(0)\right) \\
& =2 \alpha A^{\prime}(0)-n A^{\prime}(0) A^{\prime \prime}(0) \\
& -2 A^{\prime}(0)\left[n A^{\prime \prime}(0)+A^{\prime \prime}(0)\right.
\end{aligned}
$$

Using initial condition in Eq. (7) gives

$$
\begin{gathered}
A^{\prime \prime \prime}(0)=0, A^{(5)}(0)=0 \\
A^{(4)}(0)=\frac{3 \alpha^{2} A(0)}{(n+1)(n+3)\left(K_{m}+A(0)\right)^{3}}
\end{gathered}
$$

$$
=\frac{15 \alpha^{3} A(0) K_{m}\left[(n+1) K_{m}-2 A(0)(n+3)\right]}{(n+1)^{2}(n+3)(n+5)\left(K_{m}+A(0)\right)^{5}}
$$

Substituting (A2) and (A6-A9) into (A1) gives the explicit concentration form.

Substituting $x=1$ in the derived concentration and its derivative gives

$$
\begin{aligned}
& =A(0)+\frac{\alpha(1)}{(n+1)\left(K_{m}+A(0)\right)} \frac{1}{2 !} \\
& +\frac{3 \alpha^{2} A(0)}{(n+1)(n+3)\left(K_{m}+A(0)\right)^{3}} \frac{1}{4 !} \\
& +\frac{15 \alpha^{3} A(0) K_{m}\left[(n+1) K_{m}-2 A(0)(n+3)\right]}{(n+1)^{2}(n+3)(n+5)\left(K_{m}+A(0)\right)^{5}} \frac{1}{6 !} \\
& =A(0)+\frac{\alpha A(0)}{(n+1)\left(K_{m}+A(0)\right)} \frac{2}{2 !} \\
& +\frac{12 \alpha^{2} A(0)}{(n+1)(n+3)\left(K_{m}+A(0)\right)^{3}} \frac{1}{4 !} \\
& +\frac{90 \alpha^{3} A(0) K_{m}\left[(n+1) K_{m}-2 A(0)(n+3)\right]}{(n+1)^{2}(n+3)(n+5)\left(K_{m}+A(0)\right)^{5}} \frac{1}{6 !}
\end{aligned}
$$

where, $A(0)$ can be obtained from boundary condition (6). Now Eqs. (A10) and (A11) can be used in the boundary condition

$$
A^{\prime(1)}=S_{h}(1-A(1))
$$

From Eq. (A10), we can obtain the value of $A(0)$ and hence. we can obtain the concentration of substrate for slap, spherical, and cylindrical cases by using $n=0,1$, and 2 , respectively.

\section{APPENDIX B. LIMITING CASES}

\section{B.1 Saturated (zero order) catalytic kinetics}

We initially consider the situation where the substrate concentration of oxygen $A(x)$ is much greater than the Michaelis constant $K_{m}$, that is, $A(x)>>K_{m}$. In such a case, Eq. 
(5) takes on the simple linear form

$$
x A^{\prime \prime}(x)+n A^{\prime}(x)-\alpha x=0
$$

The boundary conditions are

$$
\begin{gathered}
x=0, \frac{d A}{d x}=0 \\
x=1, \frac{d A}{d x}=S_{h}(1-A)
\end{gathered}
$$

The solution is readily obtained for the following cases:

For $n=0$, Eq. (B1) becomes

$$
A^{\prime \prime}(x)-\alpha=0
$$

In this case the concentration and the effectiveness factor are, respectively,

$$
\begin{aligned}
& A(x)=\frac{1}{2}\left(\frac{2 S_{h}-\alpha\left(2+S_{h}\right)}{S_{h}}+\alpha x^{2}\right) \\
& E f=\frac{\left(1+K_{m}\right)}{\alpha}\left[\frac{d A}{d x}\right]_{x=1}=\frac{1}{\alpha}(\alpha)=1
\end{aligned}
$$

For $n=1$, Eq. (B1) becomes

$$
x A^{\prime \prime}(x)+A^{\prime}(x)-\alpha x=0
$$

and in this case, the concentration and the effictiveness factor are, respectively,

$$
\begin{aligned}
& A(x)=\frac{1}{4}\left(\frac{4 S_{h}-\alpha\left(2+S_{h}\right)}{S_{h}}+\alpha x^{2}\right) \\
& E f=\frac{\left(1+K_{m}\right)}{\alpha}\left[\frac{d A}{d x}\right]_{x=1}=\frac{1}{\alpha}\left(\frac{\alpha}{2}\right)=\frac{1}{2}
\end{aligned}
$$

For $n=2$, Eq. (B4) becomes

$$
x A^{\prime \prime}(x)+2 A^{\prime}(x)-\alpha x=0
$$

In this case, the concentration and the effictiveness factor are, respectively,

$$
\begin{aligned}
& A(x)=\frac{1}{6}\left(\frac{6 S_{h}-\alpha\left(2+S_{h}\right)}{S_{h}}+\alpha x^{2}\right) \\
& E f=\frac{\left(1+K_{m}\right)}{\alpha}\left[\frac{d A}{d x}\right]_{x=1}=\frac{1}{\alpha}\left(\frac{\alpha}{3}\right)=\frac{1}{3}
\end{aligned}
$$

\section{B.2 Unsaturated (first order) catalytic kinetics}

Here we consider the case when the substrate concentration in the film is less than the Michaelis constant. That is, we assume that $A(x)<<1$. In this case, Eq. (5) can be written as follows

$$
x A^{\prime \prime}(x)+n A^{\prime}(x)-\frac{\alpha x A(x)}{K_{m}}=0
$$

Assume a solution of the form

$$
A(x)=B_{1} \cosh (m x)+B_{2} \sinh (m x)
$$

By using boundary conditions (B2) and (B3), we obtain

$$
B_{1}=\frac{S_{h}}{m \sinh m+S_{h} \cosh m}, B_{2}=0
$$

and hence,

$$
\begin{aligned}
A(x) & =\frac{S_{h} \cosh (m x)}{m \sinh m+S_{h} \cosh m} \\
A^{\prime}(x) & =\frac{m S_{h} \sinh (m x)}{m \sinh m+S_{h} \cosh m} \\
A^{\prime \prime}(x) & =\frac{m^{2} S_{h} \cosh (m x)}{m \sinh m+S_{h} \cosh m}
\end{aligned}
$$

If we substitute these into Eq. (B14), we have

$$
\begin{array}{r}
\frac{m^{2} S_{h} \cosh (m x)}{m \sinh m+S_{h} \cosh m}+\frac{n}{x} \frac{m S_{h} \sinh (m x)}{m \sinh m+S_{h} \cosh m} \\
-\frac{\alpha}{K_{m}} \frac{m^{2} S_{h} \cosh (m x)}{m \sinh m+S_{h} \cosh m}=0
\end{array}
$$

Using L'Hospital's rule in Eq. (B21) at $x=0$ leads to

$$
\begin{aligned}
& \frac{m^{2}(1+n) S_{h} \cosh (m x)}{m \sinh m+S_{h} \cosh m} \\
& -\frac{\alpha}{K_{m}} \frac{S_{h} \cosh (m x)}{m \sinh m+S_{h} \cosh m}=0 \\
& m^{2}(1+n)-\frac{\alpha}{K_{m}}=0
\end{aligned}
$$

which implies

$$
m= \pm \sqrt{\frac{\alpha}{K_{m}(1+n)}}
$$

Substituting this value in (B16) gives the explicit concentration of the substrate. The effictive factor is given by

$$
E f=\frac{\left(1+K_{m}\right)}{\alpha} \frac{m S_{h} \sinh (m x)}{m \sinh m+S_{h} \cosh m}
$$

